\title{
Receiver Model for Depolarized Signal Due to Polarization-Mode Dispersion and Partially Polarized Noise Due to Polarization-Dependent Loss in an Optical Fiber Communication System
}

\author{
Hua Jiao, Member, IEEE, John Zweck, Li Yan, Member, IEEE, Curtis R. Menyuk, Fellow, IEEE, Fellow, OSA,
} and Gary M. Carter, Fellow, IEEE

\begin{abstract}
We systematically investigate the combined effect on the system performance in an optical fiber communication system of a signal that is depolarized due to polarization-mode dispersion (PMD) and noise that is partially polarized due to polarization-dependent loss. We derive a formula for the variance of the electric current of the signal due to the signal-noise beating between a depolarized signal and partially polarized noise. We validate this theoretical formula by comparing the $Q$-factor calculated using the theory to results obtained from Monte Carlo simulations and experiments. We show that the system performance strongly depends on the power-splitting ratio, the degree of polarization of the noise, and the angle between the states of polarization of the signal and the polarized part of the noise. Although the theoretical formula is derived assuming that the optical fiber only has first-order PMD, we show that for arbitrary fiber, this formula still produces a reliable estimate of the $Q$-factor provided that the second-order PMD is on the order of $300 \mathrm{ps}^{2}$ or less.
\end{abstract}

Index Terms-Optical fiber communication, optical fiber polarization, optical noise, optical receivers, $Q$-factor.

\section{INTRODUCTION}

$\mathbf{T}$ HE performance of optical fiber communications systems can be significantly impaired by polarization effects, such as the polarization-mode dispersion (PMD) and random variation of the birefringence in optical fibers, and the polarization-dependent loss (PDL) in devices, such as optical amplifiers [1], [2]. The interaction that the PDL has with the random fiber birefringence and with the PMD has two distinct effects on the system performance: variations in the optical SNR (OSNR) and waveform distortions.

Manuscript received August 04, 2008; revised January 28, 2009. First published May 08, 2009; current version published August 21, 2009.

H. Jiao was with the Department of Computer Science and Electrical Engineering, University of Maryland, Baltimore, MD 21250 USA. He is now with Ciena, Linthicum, MD 21090 USA (e-mail: hjiao@ciena.com).

J. Zweck is with the Department of Mathematics and Statistics, University of Maryland Baltimore County, Baltimore, MD 21250 USA (e-mail: zweck@umbc.edu).

L. Yan, C. R. Menyuk, and G. M. Carter are with the Department of Computer Science and Electrical Engineering, University of Maryland Baltimore County, Baltimore, MD 21250 USA (e-mail: liyan@umbc.edu; menyuk@umbc.edu; carter@umbc.edu).

Color versions of one or more of the figures in this paper are available online at http://ieeexplore.ieee.org.

Digital Object Identifier 10.1109/JLT.2009.2022510
Variations in the OSNR arise when initially unpolarized noise is partially polarized by the PDL and the alignment between the polarization states of a polarized signal and the polarized part of the noise is randomized due to variations in the fiber birefringence [3]-[8]. In addition, in wavelength-division multiplexed (WDM) systems, the combination of the PMD-induced walk-off between the polarization states of the signal and noise in each channel, the PDL, and the gain saturation of optical amplifiers induces random variations of the power levels of the signal and noise in each channel [9].

Waveform distortions are a well-known consequence of PMD. Since PMD also depolarizes the signal, these distortions can be more complicated in the presence of PDL. Huttner et al. [10] discuss three simple examples that illustrate the peculiar effects that can arise when a PDL element is sandwiched between two fibers with PMD. They also demonstrated that in a system with distributed PMD and PDL, the additional waveform distortion due to the interaction of the PMD and PDL can increase the bit-error ratio (BER) above the values obtained in the absence of the PDL. However, for these simulations, they used a large average PDL value of $11 \mathrm{~dB}$.

In a more recent simulation study, Shtaif and Rosenberg [11] showed that in systems with realistic parameters, the waveform distorting effects of PDL are of very limited significance, both compared to those due to PMD alone and due to the significance of PDL as a mechanism that affects the OSNR. They showed that these conclusions hold provided that the average PDL did not exceed $5 \mathrm{~dB}$. Their results were based on calculations of the eye closure penalty obtained using noise-free simulations for a $10 \mathrm{~Gb} / \mathrm{s}$ system with $50 \%$ duty cycle return-to-zero (RZ) pulses and average differential group delay (DGD) values ranging from 10 to 40 ps. However, Kim et al. [12] found that even small values of PDL (less than $1 \mathrm{~dB}$ ) can reduce the effectiveness of optical PMD compensators.

Recent research has focused on the problem of developing analytical receiver models to quantify how the BER (or equivalently, the $Q$-factor) depends on interactions between the PMD and PDL [13]-[16]. The BER can, of course, be approximated using brute-force Monte Carlo simulations. However, analytical approaches to computing the BER are also useful since they may result in greater physical insight and are often computationally less expensive than Monte Carlo simulations. 
There are two basic approaches that are commonly used to analytically calculate the BER. The first approach involves deriving an exact formula for the probability density function (pdf) of the received current in each bit [17]-[19]. The BER is then obtained from these pdfs using the method described in [20]. In the second approach, formulae are derived for the mean and standard deviation of the received current from which Gaussian approximations to the electrical pdfs are obtained [20], [21]. Even though the pdfs are not Gaussian, simulations have shown that for amplitude-shift-keyed modulation formats, the BER values obtained using the Gaussian pdfs are accurate to within a factor of 2 [22], [23].

These approaches were both initially developed for polarized signals and noise that is either unpolarized or copolarized with the signal. Both approaches have been extended to deal with the case that the signal is depolarized due to PMD and the noise is unpolarized [15], [24], [25]. The case that the noise is partially polarized and the signal is polarized was first treated by Lima et al. [13] using the second approach and validated by comparison with Monte Carlo simulations and experiments [5], [13]. Subsequently, Rebola and Cartaxo [14] used the first approach to obtain the exact pdfs. In earlier related work, Mecozzi and Shtaif [26] derived a formula for the probability distribution of the OSNR due to PDL and random birefringence.

More recently, Zhang et al. [16] used simulations to study a simplified system in which partially polarized noise was added to a signal that was degraded by the concatenation of first-order PMD and a PDL element. They derived an exact formula for the BER using the approach of [19], which they validated using Monte Carlo simulations. They used this formula to study the effect that partially polarized noise has on the BER for amplitude-shift-keyed and differential phase-shift-keyed modulation formats with and without PMD. However, they did not perform an experimental validation of the formula.

In this paper, we investigate the combined effect on the system performance due to a signal that has been depolarized due to first-order PMD and noise that has been partially polarized due to PDL. Building on the work of Lima et al. [13] and Pfennigbauer et al. [22], we derive formulae for the mean and variance of the received electric current in each bit, from which we estimate the $Q$-factor and the BER. As such, the analytical formulae we derive using the second approach to receiver modeling are complementary to the formulae that Zhang et al. [16] obtained using the first approach. We validate our results by comparing $Q$-factors obtained from the model to those obtained from Monte Carlo simulations and experiments. One of the main contributions of this paper is to provide the first experimental validation of a formula for the $Q$-factor for systems with partially polarized noise and a signal that is impaired by PMD.

In this paper, we ignore the effects of the PMD on the noise and the PDL on the signal. While it is quite reasonable to ignore the effect of the PMD on the noise, our decision to ignore the effect of the PDL on the signal requires some justification. There are three reasons for making this simplification. First, the work of Shtaif and Rosenberg [11] discussed before shows that the PDL has very little effect on the waveform distortion in realistic systems with distributed PMD and PDL. Second, since one of our main goals was to provide an experimental validation of the theory, it was important to design an experimental system in which the parameters could be measured and controlled with sufficient accuracy to perform such a validation. Therefore, we chose to devise the simplest system we could that had not been studied in previous experimental work and to develop the theory for this system. In this respect, we were inspired by the simple examples of Huttner et al. [10]. Third, to correctly incorporate the effect of the PDL on the signal would require a transmission model with both distributed PMD and PDL, together with a stochastic approach that accounted for the random variations in the fiber birefringence. While such a model could in principle be derived, it is not clear whether it would yield much additional physical insight. Moreover, because of the increased complexity of such a model, it may actually be more worthwhile to simply perform Monte Carlo simulations in this most general case.

Because higher order PMD in the fibers of an optical fiber communication system can also affect the system performance, we used Monte Carlo simulations to investigate the limitations of the theoretical formula in the presence of higher order PMD. For the limited but representative number of fiber realizations we simulated, we found that the absolute error in the theoretically computed $Q$-factor is less than 0.5 provided that the second-order PMD (SOPMD) does not exceed about $300 \mathrm{ps}^{2}$.

In Section II, we describe the idealized system setup we used to study the combined effects on system performance of PMD on the signal and PDL on the noise. Then, we derive the theoretical formula for the signal-noise beating variance. The derivation of this formula is a generalization of that given in [13] in the case of a polarized signal and partially polarized noise. In Section III, we present the results of simulations in which we validate the theory by comparison to Monte Carlo simulations and study the dependence of the $Q$-factor on the parameters of the signal, fiber, and noise. In Section IV, we describe the experiments we performed to further validate the theory. Finally, in Section V, we discuss the limitations of the theory when it is applied to signals with higher order PMD.

\section{THEORY}

To study the combined effect of PMD and PDL on the system performance, we consider the setup shown in Fig. 1. We use a piece of polarization-maintaining (PM) fiber dominated by firstorder PMD to emulate the effect of PMD on the signal during transmission. Second-order and higher order PMD effects are ignored. Although random mode coupling in single-mode fibers causes the PMD to be much more complicated in optical fiber transmission systems than in PM fiber, the use of PM fiber enables us to more readily derive a formula for the $Q$-factor that accounts for distortions induced by first-order PMD. This research can be used as a benchmark for further investigations using single-mode fibers. The power-splitting ratio, which determines the fraction of the signal power that is coupled into each of the two principal polarization modes, is controlled by a polarization controller (PC) at the input to the PM fiber. We use a source of partially polarized noise to emulate the effect on the noise due to the PDL elements during transmission. This noise source is characterized by the degree of polarization (DOP) of the noise and the state of polarization (SOP) of the polarized 


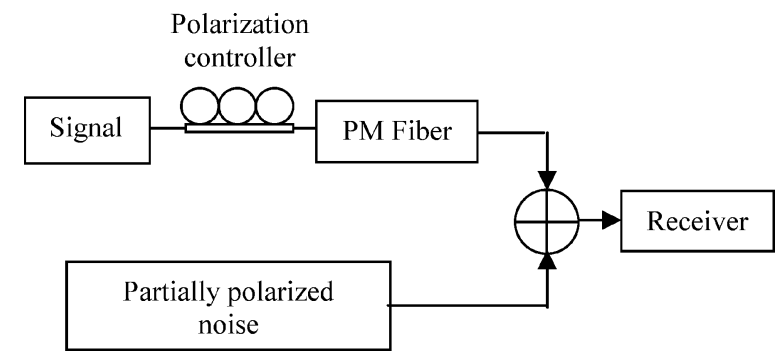

Fig. 1. System setup for receiver modeling with combined effects of PMD and PDL during transmission.

part of the noise. We ignore the effects of PDL on the signal and PMD on the noise. We also ignore other transmission effects such as chromatic dispersion and nonlinearities. The noise-free signal is combined with the partially polarized noise before entering the receiver. The receiver consists of an optical filter, a square-law photodetector, and an electrical filter. We evaluate the system performance using the $Q$-factor, which we calculate from the pdfs of the electric current in each of the ONEs and ZEROs as in [20]. We approximate each of these pdfs by Gaussians, which we calculate from the mean and variance of the electric current in each bit [20].

To avoid confusion, we now clarify the notational conventions we use for vectors. For Jones vectors with two components, we use lower case bold symbols without arrows, e.g., $\mathbf{v}$. Jones vectors $\mathbf{v}$ will always be column vectors. We let $\mathbf{v}^{\dagger}$ be the row vector that is the complex-conjugate and transpose of $\mathbf{v}$. Then, $\mathbf{v}^{\dagger} \mathbf{w}$ is a scalar and $\mathbf{v w} \mathbf{w}^{\dagger}$ is a $2 \times 2$ matrix. For a vector with three components, we use a capital bold symbol with an arrow over the top, e.g., $\overrightarrow{\mathbf{S}}$. For all vectors with four components, we use capital bold symbols without arrows, e.g., $\mathbf{S}$.

Our receiver model is a generalization of the model of Lima et al. [13]. Let $\mathbf{e}_{s}(t)$ and $\mathbf{e}_{n}(t)$ denote the Jones vectors of the electric field envelopes of the noise-free signal and the noise, respectively, prior to the receiver, where $t$ is the time. Let $H_{o}(\omega)$, $h_{o}(t), H_{e}(\omega)$, and $h_{e}(t)$ denote the transfer function and the corresponding impulse response of the optical and the electrical filters, respectively. The noise-free signal and the noise after the optical filter are given by convolving the impulse response of the optical filter with the electric fields of the signal and the noise, respectively, which we denote by $\mathbf{e}_{s_{o}}=\mathbf{e}_{s} * h_{o}$ and $\mathbf{e}_{n_{o}}=\mathbf{e}_{n} *$ $h_{o}$. As in [13], we assume that the scalar autocorrelation function of the optically filtered noise is given by $\left\langle\mathbf{e}_{n_{o}}^{\dagger}(t) \mathbf{e}_{n_{o}}\left(t^{\prime}\right)\right\rangle=$ $N_{\mathrm{ASE}} r_{o}\left(t^{\prime}-t\right)$, where $N_{\mathrm{ASE}}$ is the total power spectral density of the noise and $r_{o}(\tau)=\int_{-\infty}^{+\infty} h_{o}\left(\tau^{\prime}\right) \bar{h}_{o}\left(\tau+\tau^{\prime}\right) d \tau^{\prime}$ is the autocorrelation function of the optical filter. Here, $\bar{z}$ denotes the complex conjugate of a complex scalar $z$. To model partially polarized noise, we introduce the temporal coherency matrix of the optically filtered noise $J_{n}(\tau)=\left\langle\mathbf{e}_{n_{o}}(t) \mathbf{e}_{n_{o}}^{\dagger}(t+\tau)\right\rangle$. If the noise has not been affected by PMD, we may assume that the noise process is wide-sense stationary, in which case $J_{n}(\tau)=\left(r_{o}(\tau) / r_{o}(0)\right) J_{n}$, where $J_{n}=J_{n}(0)$. After the signal and the noise have passed through the optical filter, the photo- detector, and the electric filter, the electric current of the combined signal and noise is given by

$$
i=R\left|\left[\mathbf{e}_{s}+\mathbf{e}_{n}\right] * h_{o}\right|^{2} * h_{e}=i_{s}+\left\langle i_{n}\right\rangle
$$

where $i_{s}=R\left|\mathbf{e}_{s_{o}}\right|^{2} * h_{e}$ is the electric current of the noise-free signal and $\left\langle i_{n}\right\rangle(t)=\left\langle i_{n}\right\rangle=R N_{\mathrm{ASE}} B_{o}$ is the mean current of the noise after the electrical filter. Here, $R$ is the responsivity of the photodetector and $B_{o}=r_{o}(0)$ is the noise equivalent bandwidth of the optical filter. The variance of the electric current at time $t$ has the form

$$
\sigma^{2}(t)=\sigma_{\mathrm{ASE}-\mathrm{ASE}}^{2}+\sigma_{S-\mathrm{ASE}}^{2}(t) .
$$

The first term on the right-hand side of (2) is the variance of the electric current due to the beating of the noise with itself and is given by [13]

$$
\sigma_{\mathrm{ASE}-\mathrm{ASE}}^{2}=\frac{1}{2} R^{2} N_{\mathrm{ASE}}^{2} \frac{I_{\mathrm{ASE}-\mathrm{ASE}}}{\Gamma_{\mathrm{ASE}-\mathrm{ASE}}}
$$

where $\Gamma_{\mathrm{ASE}}$-ASE $=1 /\left(1+\mathrm{DOP}_{n}^{2}\right)$ is the noise-noise beating factor and $I_{\mathrm{ASE}-\mathrm{ASE}}=\int_{-\infty}^{+\infty}\left|r_{o}(\tau)\right|^{2} r_{e}(\tau) d \tau$. Here, $\mathrm{DOP}_{n}$ is the DOP of the partially polarized noise and $r_{e}(\tau)$ is the autocorrelation function of the electrical filter, which is defined by $r_{e}(\tau)=\int_{-\infty}^{+\infty} \bar{h}_{e}\left(\tau^{\prime}\right) h_{e}\left(\tau+\tau^{\prime}\right) d \tau^{\prime}$. The formulae for the average current in the marks $\left\langle i_{1}\right\rangle$, the average current in the spaces $\left\langle i_{0}\right\rangle$, and the noise-noise beating variance $\sigma_{\mathrm{ASE}}^{2} \mathrm{ASE}$ in (1) and (3) are the same for a depolarized signal with partially polarized noise as for a polarized signal. The only change is to $\sigma_{S-\mathrm{ASE}^{2}}(t)$ in (2).

We now derive a formula for the variance $\sigma_{S-\mathrm{ASE}}^{2}(t)$ due to the beating between a depolarized signal and partially polarized noise. Let $u(t)$ be the scalar electric field of the optical signal exiting the transmitter. After passing through a fiber with only first-order PMD, we can write the electric field of the noise-free signal as

$$
\mathbf{e}_{s}(t)=c_{+} u\left(t-\frac{\tau}{2}\right) \mathbf{v}_{+}+c_{-} u\left(t+\frac{\tau}{2}\right) \mathbf{v}_{-}
$$

where $\mathbf{v}_{ \pm}$are unit vectors that represent the two principal states of the fiber in Jones space, $\tau$ is the DGD, and $\left|c_{+}\right|^{2}+\left|c_{-}\right|^{2}=1$. Therefore, $\left|c_{+}\right|^{2}$ is the power-splitting ratio, which represents the fraction of the total signal power that is coupled to the principal mode $\mathbf{v}_{+}$. For simplicity of notation, let $U=u * h_{o}$. After the optical filter, the electric field of the signal is given by

$$
\mathbf{e}_{s_{o}}(t)=c_{+} U\left(t-\frac{\tau}{2}\right) \mathbf{v}_{+}+c_{-} U\left(t+\frac{\tau}{2}\right) \mathbf{v}_{-} .
$$

Let $\sum=\left(\sigma_{0}, \sigma_{3}, \sigma_{1},-\sigma_{2}\right)^{\mathrm{T}}$ be the column vector whose entries are the Pauli spin matrices [27], where

$\boldsymbol{\sigma}_{0}=\left(\begin{array}{ll}1 & 0 \\ 0 & 1\end{array}\right), \boldsymbol{\sigma}_{1}=\left(\begin{array}{ll}0 & 1 \\ 1 & 0\end{array}\right), \boldsymbol{\sigma}_{2}=\left(\begin{array}{cc}0 & -i \\ i & 0\end{array}\right), \boldsymbol{\sigma}_{3}=\left(\begin{array}{cc}1 & 0 \\ 0 & -1\end{array}\right)$.

Motivated by [15, eqs. (3.15)-(3.17)], we define the two-time Stokes parameters of the signal at times $t$ and $s$ by

$$
S_{s}(t, s)=e_{s_{0}}^{\dagger}(t) \sum e_{s_{0}}(s)
$$


i.e.,

$$
\mathbf{S}_{s}(t, s)=\left(\begin{array}{c}
S_{0}(t, s) \\
\overrightarrow{\mathbf{S}}_{s}(t, s)
\end{array}\right)
$$

with

$$
\overrightarrow{\mathbf{S}}(t, s)=\left(S_{1}(t, s), S_{2}(t, s), S_{3}(t, s)\right)^{\mathrm{T}}
$$

where

$$
\begin{aligned}
& S_{0}(t, s)=\mathbf{e}_{s_{o}}^{\dagger}(t) \mathbf{e}_{s_{o}}(s) \\
& S_{1}(t, s)=\mathbf{e}_{s_{o}}^{\dagger}(t) \boldsymbol{\sigma}_{3} \mathbf{e}_{s_{o}}(s) \\
& S_{2}(t, s)=\mathbf{e}_{s_{o}}^{\dagger}(t) \boldsymbol{\sigma}_{1} \mathbf{e}_{s_{o}}(s) \\
& S_{3}(t, s)=-\mathbf{e}_{s_{o}}^{\dagger}(t) \boldsymbol{\sigma}_{2} \mathbf{e}_{s_{o}}(s) .
\end{aligned}
$$

The two-time Stokes parameters can be written as

$$
\begin{aligned}
\mathbf{S}(t, s)= & \left|c_{+}\right|^{2} \bar{U}\left(t-\frac{\tau}{2}\right) U\left(s-\frac{\tau}{2}\right) \mathbf{S}_{++} \\
& +\left|c_{-}\right|^{2} \bar{U}\left(t+\frac{\tau}{2}\right) U\left(s+\frac{\tau}{2}\right) \mathbf{S}_{--} \\
& +\bar{c}_{+} c_{-} \bar{U}\left(t-\frac{\tau}{2}\right) U\left(s+\frac{\tau}{2}\right) \mathbf{S}_{+-} \\
& +\bar{c}_{-} c_{+} \bar{U}\left(t+\frac{\tau}{2}\right) U\left(s-\frac{\tau}{2}\right) \mathbf{S}_{-+}
\end{aligned}
$$

where

$$
\begin{array}{ll}
\mathbf{S}_{++}=\mathbf{v}_{+}^{\dagger} \sum \mathbf{v}_{+} & \mathbf{S}_{--}=\mathbf{v}_{-}^{\dagger} \sum \mathbf{v}_{-} \\
\mathbf{S}_{+-}=\mathbf{v}_{+}^{\dagger} \sum \mathbf{v}_{-} & \mathbf{S}_{-+}=\mathbf{v}_{-}^{\dagger} \sum \mathbf{v}_{+} .
\end{array}
$$

Note that $\mathbf{S}_{++}$and $\mathbf{S}_{--}$are real vectors whereas $\mathbf{S}_{+-}$and $\mathbf{S}_{-+}$are complex vectors, with $\overline{\mathbf{S}}_{+-}=\mathbf{S}_{-+}$. The four-component Stokes parameters of the noise are represented by

$$
\mathbf{S}^{N}=\left(\begin{array}{c}
S_{0}^{N} \\
\overrightarrow{\mathbf{S}}^{N}
\end{array}\right)
$$

with $\overrightarrow{\mathbf{S}}^{N}=\left(S_{1}^{N}, S_{2}^{N}, S_{3}^{N}\right)^{\mathrm{T}}$. Since the noise process is wide-sense stationary, the variance of the electric current due to signal-noise beating may be expressed as

$$
\sigma_{S-\mathrm{ASE}}^{2}(t)=R^{2} \mathbf{S}^{N} \cdot \mathbf{I}_{S-\mathrm{ASE}}(t)
$$

where $\mathbf{S} \cdot \mathbf{I}$ denotes the standard inner product of real four vectors. Here,

$$
\begin{aligned}
& \mathbf{I}_{S-\operatorname{ASE}}(t)=\left(\begin{array}{c}
I_{S-\mathrm{ASE}^{0}}^{(t)} \\
\overrightarrow{\mathbf{I}}_{S-\mathrm{ASE}^{(t)}}
\end{array}\right)= \\
& \int_{-\infty}^{\infty} \int_{-\infty}^{\infty} \mathbf{S}_{s}(w, z) r_{o}(z-w) h_{e}(t-w) h_{e}(t-z) d w d z
\end{aligned}
$$

and

$$
\mathbf{S}^{N}=N_{\mathrm{ASE}}\left(\begin{array}{c}
1 \\
\operatorname{DOP}_{n} \overrightarrow{\mathbf{S}}_{N}^{(p)}
\end{array}\right)
$$

where $\overrightarrow{\mathbf{S}}_{N}^{(P)}=\overrightarrow{\mathbf{S}}^{N} /\left|\overrightarrow{\mathbf{S}}^{N}\right|$ is the unit Stokes vector of the polarized part of the noise. By (8)-(10), we have

$$
\sigma_{S-\mathrm{ASE}}^{2}(t)=R^{2} N_{\mathrm{ASE}}\left[I_{S-\mathrm{ASE}}^{0}(t)+\mathrm{DOP}_{n} \overrightarrow{\mathbf{I}}_{S-\mathrm{ASE}}(t) \cdot \overrightarrow{\mathbf{S}}_{N}^{(p)}\right] .
$$

Next, we define

$$
\begin{aligned}
& I_{S-\mathrm{ASE}}(t) \\
& =2 \int_{-\infty}^{\infty} \int_{-\infty}^{\infty} \bar{U}(w) U(z) r_{o}(z-w) h_{e}(t-z) h_{e}(t-w) d w d z \\
& I_{S-\mathrm{ASE}}(t ; \tau) \\
& =2 \int_{-\infty}^{\infty} \int_{-\infty}^{\infty} \bar{U}(w) U(z+\tau) r_{o}(z-w) h_{e}(t-w) h_{e}(t-z) d w d z .
\end{aligned}
$$

Then, by (6), (9), (12), and (13), we have

$$
\begin{aligned}
\mathbf{I}_{S-\operatorname{ASE}}(t)= & \frac{1}{2}\left|c_{+}\right|^{2} I_{S-\mathrm{ASE}}\left(t-\frac{\tau}{2}\right) \mathbf{S}_{++} \\
& +\frac{1}{2}\left|c_{-}\right|^{2} I_{S-\mathrm{ASE}}\left(t+\frac{\tau}{2}\right) \mathbf{S}_{--} \\
& +\operatorname{Re}\left[\bar{c}_{+} c_{-} I_{S-\mathrm{ASE}}\left(t-\frac{\tau}{2} ; \tau\right) \mathbf{S}_{+-}\right]
\end{aligned}
$$

Since $\left|\mathbf{v}_{ \pm}\right|=1$, we have that $\mathbf{S}_{++}^{0}=\mathbf{S}_{--}^{0}=1$ and $\mathbf{S}_{+-}^{0}=$ 0 . Since we neglect the effect of PDL on the signal, we have $\overrightarrow{\mathbf{S}}_{--}=-\overrightarrow{\mathbf{S}}_{++}$. Therefore, by (8), the variance of the electric current due to the beating between the depolarized signal and the partially polarized noise can be expressed as

$$
\begin{aligned}
& \sigma_{S-\mathrm{ASE}}^{2}(t) \\
&=\frac{1}{2} R^{2} N_{\mathrm{ASE}}\left\{\left|c_{+}\right|^{2} I_{S-\mathrm{ASE}}\left(t-\frac{\tau}{2}\right)+\left|c_{-}\right|^{2} I_{S-\mathrm{ASE}}\left(t+\frac{\tau}{2}\right)\right. \\
& \\
&+\operatorname{DOP}_{n} \overrightarrow{\mathbf{S}}_{N}^{(p)} \cdot\left[\left(\left|c_{+}\right|^{2} I_{S-\mathrm{ASE}}\left(t-\frac{\tau}{2}\right)\right.\right. \\
&\left.-\left|c_{-}\right|^{2} I_{S-\mathrm{ASE}}\left(t+\frac{\tau}{2}\right)\right) \overrightarrow{\mathbf{S}}_{++} \\
&\left.\left.+2 \operatorname{Re}\left(\bar{c}_{+} c_{-} I_{S-\mathrm{ASE}}\left(t-\frac{\tau}{2} ; \tau\right) \overrightarrow{\mathbf{S}}_{+-}\right)\right]\right\} .
\end{aligned}
$$

From (15), we can see that the variance due to signal-noise beating is determined by the power-splitting ratio, the DGD of the fiber, the DOP of the noise, the angle between the polarized part of the noise and the principal state vectors, and the relative phases between $c_{+}, c_{-}$, and $I_{S-A S E}(t-\tau / 2 ; \tau)$. To compare (15) to experimental results, we need to relate the quantities involved in (15) to experimentally measurable parameters. To do so, we define the average Stokes vector of the signal $\mathbf{e}_{s_{o}}(t)$ by

$$
\overrightarrow{\mathbf{S}}_{s}=\int \overrightarrow{\mathbf{S}}_{s}(t, t) d t
$$

and let

$$
P_{\tau}=\int \overline{U(t)} U(t+\tau) d t
$$


be the autocorrelation function of the input signal $U$. From (5), (16), and (17), we have

$$
\begin{aligned}
\left|c_{+}\right|^{2} & =\frac{1}{2}\left(1+\frac{\overrightarrow{\mathbf{S}}_{s} \cdot \overrightarrow{\mathbf{S}}_{++}}{P_{0}}\right) \\
2 \Re\left(\bar{c}_{+} c_{-} P_{\tau}\right) & =\frac{\overrightarrow{\mathbf{S}}_{s} \cdot \Re\left(\overrightarrow{\mathbf{S}}_{+-}\right)}{\left|\Re\left(\overrightarrow{\mathbf{S}}_{+-}\right)\right|^{2}} \\
2 \Im\left(\bar{c}_{+} c_{-} P_{\tau}\right) & =-\frac{\overrightarrow{\mathbf{S}}_{s} \cdot \Im\left(\overrightarrow{\mathbf{S}}_{+-}\right)}{\left|\Im\left(\overrightarrow{\mathbf{S}}_{+-}\right)\right|^{2}} .
\end{aligned}
$$

To extract $\Re\left(\bar{c}_{+} c_{-}\right)$and $\Im\left(\bar{c}_{+} c_{-}\right)$from (19) and (20), we use matrix inversion to obtain

$$
\left(\begin{array}{c}
\Re\left(\bar{c}_{+} c_{-}\right) \\
\Im\left(\bar{c}_{+} c_{-}\right)
\end{array}\right)=\frac{1}{\left|P_{\tau}\right|^{2}}\left(\begin{array}{cc}
\Re\left(P_{\tau}\right) & \Im\left(P_{\tau}\right) \\
-\Im\left(P_{\tau}\right) & \Re\left(P_{\tau}\right)
\end{array}\right)\left(\begin{array}{c}
\Re\left(\bar{c}_{+} c_{-} P_{\tau}\right) \\
\Im\left(\bar{c}_{+} c_{-} P_{\tau}\right)
\end{array}\right) .
$$

From (7), the Jones vectors of the principal states of the fiber $\mathbf{v}_{+}$and $\mathbf{v}_{-}$can be calculated from $\mathbf{S}_{++}$and $\mathbf{S}_{--}$, which can be measured directly in the experiment. From $\mathbf{v}_{+}$and $\mathbf{v}_{-}$, we can calculate $\mathbf{S}_{+-}$and $\mathbf{S}_{-+}$using (7). Therefore, (15) can be validated experimentally by measuring the pulse shape $u$ of the signal at the transmitter, $\overrightarrow{\mathbf{S}}_{s}, \overrightarrow{\mathbf{S}}_{N}^{(p)}, \mathrm{DOP}_{n}$, and the shapes of the optical and electrical filters.

\section{Simulations}

In this section, we perform simulations to validate formula (15) by comparison with Monte Carlo simulations and to quantify the degree to which the $Q$-factor varies as the angle between the signal and noise is varied on the Poincare sphere.

We simulated $10 \mathrm{~Gb} / \mathrm{s}$ signal transmission using a piece of PM fiber, as shown in Fig. 1. At the transmitter, the carrier wavelength was $1554.0 \mathrm{~nm}$. We used an RZ modulation format with a Gaussian pulse that had a full-width half-maximum (FWHM) width of 23 ps. The extinction ratio of the pulse was $-15 \mathrm{~dB}$, and there was no chirp in the signal. We used a pseudorandom bit string with a string length of 8 bits. The average power of the signal was $0 \mathrm{dBm}$ and the OSNR was fixed at $15 \mathrm{~dB}$. The total DGD of the fiber was $36 \mathrm{ps}$ and the length of the fiber was $21 \mathrm{~m}$. We ignored the effects of chromatic dispersion and nonlinearity in this short fiber. The parameters of the fiber are the same as a piece of PM fiber that we used in the experiment, which is discussed in Section IV. For simplicity and without loss of generality, we set the two principal states of the fiber to be $\overrightarrow{\mathbf{S}}_{++}=(-1,0,0)$ and $\overrightarrow{\mathbf{S}}_{--}=(1,0,0)$. The receiver consisted of a Gaussian-shaped optical filter with an FWHM of $60 \mathrm{GHz}$, a square-law photodetector, and a fifth-order, low-pass electrical Bessel filter with a 3-dB bandwidth of $8.5 \mathrm{GHz}$.

In the simulation, the DOP of the partially polarized noise was fixed to 0.5 and the power-splitting ratio was 0.5 . To validate formula (15), we varied the angle between the SOP of the signal and the polarized part of the noise on the Poincare sphere in the following two ways, as shown in Fig. 2. We call these ways the orthogonal and parallel cases.

In both cases, the average SOP of the signal $\overrightarrow{\mathbf{S}}_{s}$ is in the $y-z$ plane since the power-splitting ratio $\left|c_{+}\right|^{2}$ is 0.5 and $\overrightarrow{\mathbf{S}}_{++}=$ $(-1,0,0)$. Therefore, the normalized average SOP of the signal is of the form $[0, \sin (\theta), \cos (\theta)]$, where $\theta$ is the angle from the $z$ axis to $\overrightarrow{\mathbf{S}}_{s}$. In the simulations, for each of several values of $\theta$,

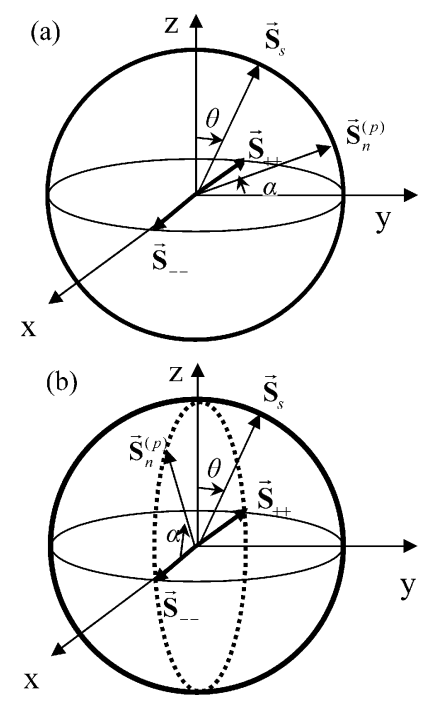

Fig. 2. Diagram of simulation setups for $\left|c_{+}\right|^{2}=0.5$. (a) Orthogonal case. (b) Parallel case.

we varied the angle between the signal and the polarized part of the noise by rotating $\overrightarrow{\mathbf{S}}_{n}^{(p)}$. In the orthogonal case shown in Fig. 2(a), let the SOP of the polarized part of the noise rotate in the $y-z$ plane, i.e., $\overrightarrow{\mathbf{S}}_{n}^{(p)}=(0, \cos (\alpha), \sin (\alpha))$, where $\alpha$ is the angle from the $y$ axis to $\overrightarrow{\mathbf{S}}_{n}^{(p)}$. We call this case the orthogonal case because the Stokes vector of the polarized part of the noise is always orthogonal to the principal state vectors. In the parallel case shown in Fig. 2(b), the SOP of the signal is the same as in the orthogonal case. However, $\overrightarrow{\mathbf{S}}_{n}^{(p)}$ rotates in the $x-z$ plane on the dotted circle. We call this case the parallel case because the two principal state vectors are always parallel to the $x-z$ plane, which is the plane in which the Stokes vector of the polarized part of the noise lies.

In Fig. 3, the $Q$-factors calculated using (15) and from Monte Carlo simulations are plotted as a function of the angle $\alpha$. For simplicity, in the Monte Carlo simulations, the $Q$-factors were calculated using the mean and variance of the worst ONEs and worst ZEROs, which are the middle ONE between two ZEROs (010) and the middle ZERO between two ONEs (101), respectively, for an 8-bit random string. However, the $Q$-factors calculated using other bits are almost the same, differing by at most 0.1 .

In Fig. 3, the lines represent the $Q$-factor calculated using (15) and the symbols are results from Monte Carlo simulations for several values of the angle $\theta$. The agreement between Monte Carlo simulations and the theory is excellent. The slight deviations between the $Q$-factors calculated using Monte Carlo simulations and those obtained from the theory are due to the limited number of 10000 noise realizations we used in the Monte Carlo simulations.

To understand the results for the orthogonal case shown in Fig. 3(a), observe that in Fig. 2(a), as $\overrightarrow{\mathbf{S}}_{n}^{(p)}$ is rotated in the $y-z$ plane, the angle between $\overrightarrow{\mathbf{S}}_{n}^{(p)}$ and $\overrightarrow{\mathbf{S}}_{s}$ varies between 0 and $2 \pi$. In the case that $\theta=\pi / 2$ and $\alpha=0, \overrightarrow{\mathbf{S}}_{n}^{(p)}$ and $\overrightarrow{\mathbf{S}}_{s}$ are parallel. So, the beating between the signal and the noise is the maximized and the $Q$-factor attains its minimum value, as shown in Fig. 3(a). When $\overrightarrow{\mathbf{S}}_{n}^{(p)}$ is rotated so that $\alpha=\pi, \overrightarrow{\mathbf{S}}_{n}^{(p)}$ and $\overrightarrow{\mathbf{S}}_{s}$ are 

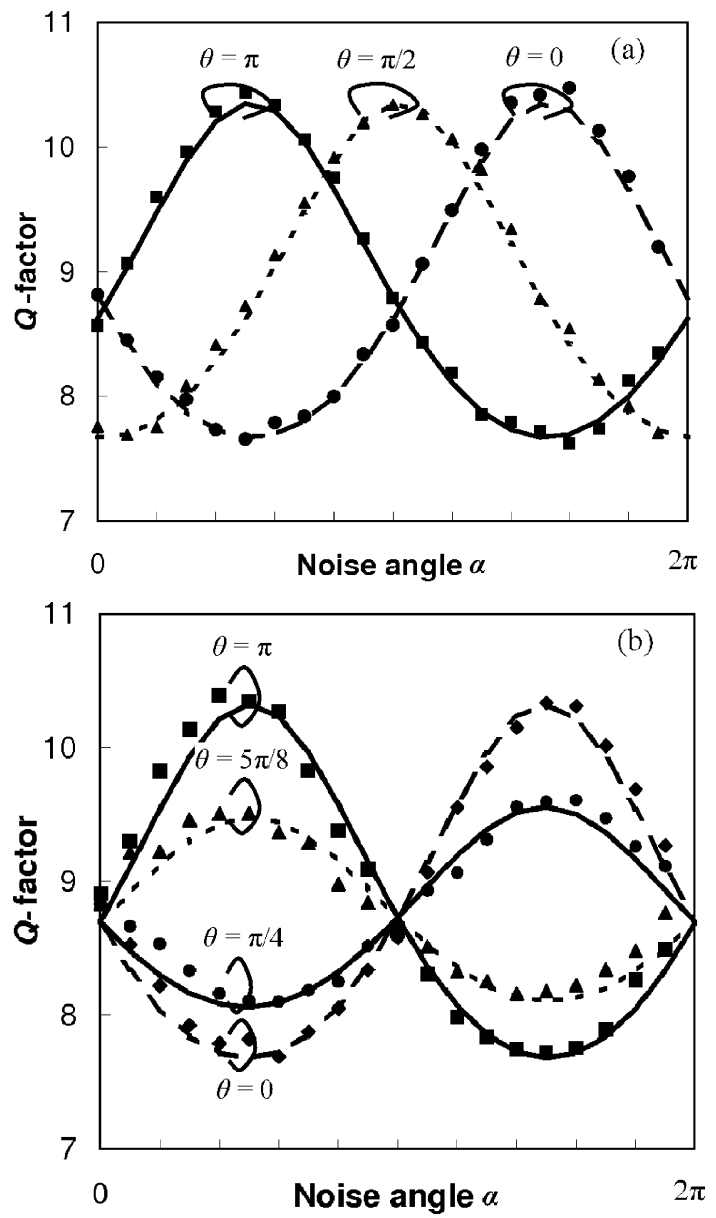

Fig. 3. $Q$-factor as a function of the angle $\alpha$ for $\left|c_{+}\right|^{2}=0.5$. The lines are calculated from formula (15) and the symbols are results from Monte Carlo simulations. (a) Orthogonal case. (b) Parallel case.

antipodal on the Poincaré sphere (orthogonal in Jones space), the beating between the signal and noise is minimized, and the $Q$-factor, therefore, attains its maximum. For other values of $\theta$ between 0 and $\pi$, the maximum and the minimum of the $Q$-factor still occurs when $\overrightarrow{\mathbf{S}}_{n}^{(p)}$ and $\overrightarrow{\mathbf{S}}_{s}$ are, respectively, parallel and antipodal to each other on the Poincare sphere, and the magnitude of the $Q$-factor variation with respect to the noise angles $\alpha$ is independent of $\theta$. Next, we provide physical interpretation of the results for the parallel case shown in Fig. 3(b).

In Fig. 2(b), $\overrightarrow{\mathbf{S}}_{s}$ is still in the $y-z$ plane but $\overrightarrow{\mathbf{S}}_{n}^{(p)}$ now rotates in the $x-z$ plane. When $\theta=0, \overrightarrow{\mathbf{S}}_{s}$ is on the $z$-axis. Thus, the beating between the signal and the noise is maximized and minimized when $\alpha$ is $\pi / 2$ and $-\pi / 2$ and the angles between $\overrightarrow{\mathbf{S}}_{n}^{(p)}$ and $\overrightarrow{\mathbf{S}}_{s}$ are 0 and $\pi$, respectively. When $\theta$ is neither 0 nor $\pi$, $\overrightarrow{\mathbf{S}}_{s}$ is not in the $x-z$ plane. In this case, no matter where $\overrightarrow{\mathbf{S}}_{n}^{(p)}$ is in the $x-z$ plane, the angle between $\overrightarrow{\mathbf{S}}_{n}^{(p)}$ and $\overrightarrow{\mathbf{S}}_{s}$ is never 0 or $\pi$. As a result, the beating between the signal and the polarized part of the noise is always partial and the $Q$-factor variation is smaller compared to the case when $\theta=0$. Quantitatively speaking, when $\theta \neq 0, \pi / 2$, or $\pi$, the minimum and maximum of the possible angles between $\overrightarrow{\mathbf{S}}_{n}^{(p)}$ and $\overrightarrow{\mathbf{S}}_{s}$ that are attainable depend on $\theta$. For example, when $0<\theta<\pi / 2$, the minimum and maximum possible angles between $\overrightarrow{\mathbf{S}}_{n}^{(p)}$ and $\overrightarrow{\mathbf{S}}_{s}$ are $\theta$ and $\pi-\theta$, respectively.

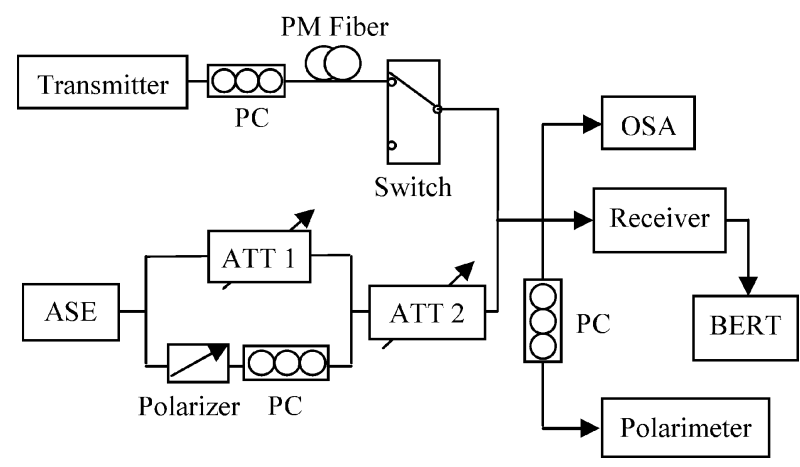

Fig. 4. Schematic diagram of the experimental setup.

\section{EXPERIMENTAL RESULTS}

In this section, we validate the receiver model described in Section II by comparison to results from experiments. The schematic diagram of the experimental setup we used is shown in Fig. 4.

The transmitter generated a $10-\mathrm{Gb} / \mathrm{s} \mathrm{RZ}$ signal with a pseudorandom pattern length of $2^{7}-1$. The FWHM width of the pulses was about $23 \mathrm{ps}$. We used a piece of PM fiber with a DGD of 36 ps to emulate the effect of PMD on the signal during transmission. SOPMD and higher order PMD were negligible in the PM fiber. The power-splitting ratio was controlled by a PC at the input of the PM fiber. We emulated the partially polarized noise in a transmission system due to PDL by combining unpolarized noise with polarized noise. The two types of noise were obtained by a 50-50 splitting of a single amplified spontaneous emission (ASE) noise source that generated unpolarized noise. The polarized noise was obtained by using a polarizer to polarize half of the unpolarized noise. A PC after the polarizer was used to control the SOP of the polarized noise. We varied the power of the other half of the noise using a tunable attenuator (ATT 1) so as to achieve a desired DOP once the unpolarized and polarized noise were combined. Finally, a second tunable attenuator (ATT 2) was used to achieve a desired OSNR at the receiver. The OSNR was fixed at $15 \mathrm{~dB}$ and monitored by an optical spectrum analyzer (OSA) with a resolution bandwidth of $0.2 \mathrm{~nm}$. We used a polarimeter to measure the SOP and DOP of the signal and the noise. The principal states of polarization of the PM fiber were calculated using Poincaré arc method [28] from measurements of the SOP of the signal at three wavelengths. To separately measure the SOP of the noise-free signal and the noise, we used a mechanical switch to turn the signal on and off and the second tunable attenuator (ATT 2) to turn the noise on and off. We used the PC at the input of the polarimeter to obtain the desired principal state of polarization (PSP) of $(0,-1,0)$ and $(0,1,0)$. Although the model and the experiment can use any PSP, we made this to enable easy monitoring of the SOP of the signal and the polarized part of the noise during the experiment. The receiver consisted of an optical filter with an FWHM of $60 \mathrm{GHz}$, a square-law photodetector, and a fifth-order, low-pass electrical Bessel filter with a 3-dB bandwidth of $9.8 \mathrm{GHz}$. The $Q$-factor was obtained from BER margin measurements [29] made using a BER tester (BERT) that has an unknown electrical bandwidth in the decision circuit and can operate at bit rates of up to $12.5 \mathrm{~Gb} / \mathrm{s}$. 
In PM fibers, the polarization modes do not vary along the fiber and energy is not exchanged between the two principal modes. More importantly, the polarization modes and the DGD are independent of wavelength. However, if the signal is not launched along either of the two principal states, then the SOP of the signal is a function of the wavelength after the signal passes through the PM fiber. In contrast to single-mode fibers with random-mode coupling, the SOP of the signal in PM fibers traces a circle on the Poincaré sphere as the wavelength is varied. The SOP of the signal rotates about the axis given by the principal state vector, and the rotation rate of the SOP of the signal as a function of wavelength is proportional to the DGD of the PM fiber. It is known that the SOP of the signal in optical fibers is sensitive to temperature and environmental perturbations and it drifts randomly over time [30]. Moreover, the drift of the SOP of the signal is faster in PM fibers than in single-mode fibers. Since the variance of the electric current of the signal due to signal-noise beating is a function of the angle between the SOP of the signal and the polarized part of the noise, it is critical to keep the SOP of the signal stable during each measurement of the $Q$-factor.

In Fig. 5, we illustrate the drift of the SOP of a monochromatic signal at the output of an 8-m PM fiber with 10 ps DGD. In Fig. 5(a), we plot the SOP of the signal $\left(\overrightarrow{\mathbf{S}}_{\mathrm{sig}}\right)$ on the Poincaré sphere that we measured over the course of an hour at the output of an 8-m PM fiber with a DGD of 10 ps. In addition to plotting the data in Fig. 5(a), we also show a circle fitted to the data using three well-spaced samples of $\overrightarrow{\mathbf{S}}_{\mathrm{sig}}$. In contrast to the random drift in single-mode fibers, we can see that in PM fibers, the SOP of the signal drifts on a circle and that this circle is orthogonal to the PSP vector. The location of the circle is determined by the PSP vector and the launching polarization states of the signal at the input of the PM fiber. In Fig. 5(b), the Stokes parameters of the signal are plotted as a function of time over a 60-min period. In Fig. 5(c), we plot the angle $\theta$ through which the SOP of the signal drifts during each 1-min interval of a 60 -min measurement period. The drift angle $\theta$ is defined to be the angle between the SOP of the signal measured at two different times. The high peaks in Fig. 5(c) were due to air-conditioning events in the laboratory that occurred every $35 \mathrm{~min}$. From Fig. 5(b) and (c), we can see that the SOP of the signal drifts quickly and it can drift up to $1 \mathrm{rad}$ within a minute. Even when the drift is slowest, the drift angle within a minute can still be as large as $0.25 \mathrm{rad}$, which corresponds to about $14^{\circ}$. Since this fast drift can induce significant measurement uncertainty in the $Q$-factor in the experiment, it has to be suppressed.

To suppress the drift rate of the SOP of the signal, we immersed the PM fiber in water to isolate the PM fiber from temperature variations and environmental perturbations. This technique proved to be very effective in reducing the drift of the SOP of the signal. In Fig. 6, we show the drift rate of the SOP of the signal after the PM fiber is put in water.

As we see in Fig. 6(c), the drift rate of the SOP of the signal during the slow-drift time windows has been reduced to less than $0.05 \mathrm{rad}$. By performing experiments in the time windows where the drift rate is low, we significantly reduced measurement error of the $Q$-factor due to the drift of the SOP of the signal. The drift of the SOP of the signal between $Q$-factor measurements has no effect on the measurement uncertainty in the $Q$-factor.

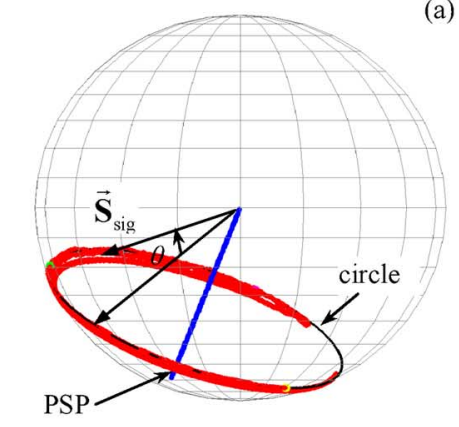

(a)
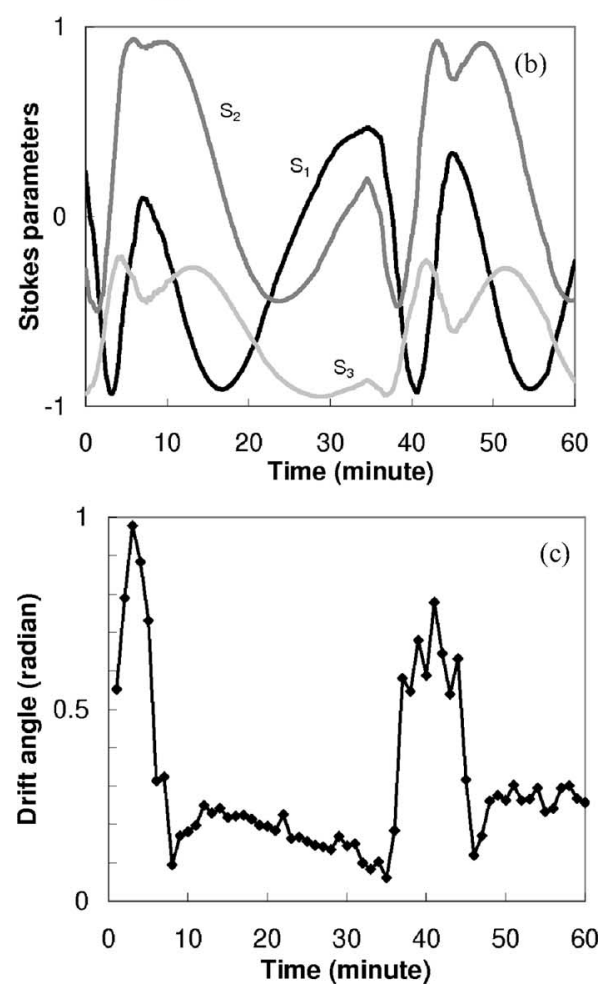

Fig. 5. Drift of the SOP of the signal over $1 \mathrm{~h}$ in an $8-\mathrm{m}$ PM fiber with $10 \mathrm{ps}$ DGD. (a) Drift of the SOP on the Poincare sphere. Here, $\overrightarrow{\mathbf{S}}_{\text {sig }}$ is the SOP of the signal. (b) Drift of the Stokes parameters of $\overrightarrow{\mathbf{S}}_{\text {sig. }}$. (c) Angle $\theta$ shown in (a) through which the SOP drifts during each 1-min interval of a 60-min measurement period.

As in the Monte Carlo simulations, we designed experiments to validate formula (15) by comparing the $Q$-factors obtained from the theory and the experiment. In the experiments, we chose two power-splitting ratios of 0 and 0.5 . For each powersplitting ratio, two levels of the DOP of the noise were used, which were 0.5 and 1.0. For each power-splitting ratio and each DOP of the noise, we varied the angle between the SOP of the signal and the polarized part of noise. For each angle, we measured the $Q$-factor and the Stokes vectors of the signal and the polarized part of the noise. We then used these measured Stokes vectors to calculate the $Q$-factor from (1)-(3) and (15) using the method described in [20].

First, we studied the case where the power-splitting ratio $\left|c_{+}\right|^{2}$ is equal to 0 by launching the signal in the principal state $\overrightarrow{\mathbf{S}}_{--}=(0,-1,0)$. In this case, the SOP of the signal $\overrightarrow{\mathbf{S}}_{s}$ was fixed and stable over time. We varied the angle $\alpha$ between $\overrightarrow{\mathbf{S}}_{s}$ and the polarized part of the noise $\overrightarrow{\mathbf{S}}_{n}^{(p)}$ by rotating $\overrightarrow{\mathbf{S}}_{n}^{(p)}$ in $45^{\circ}$ 

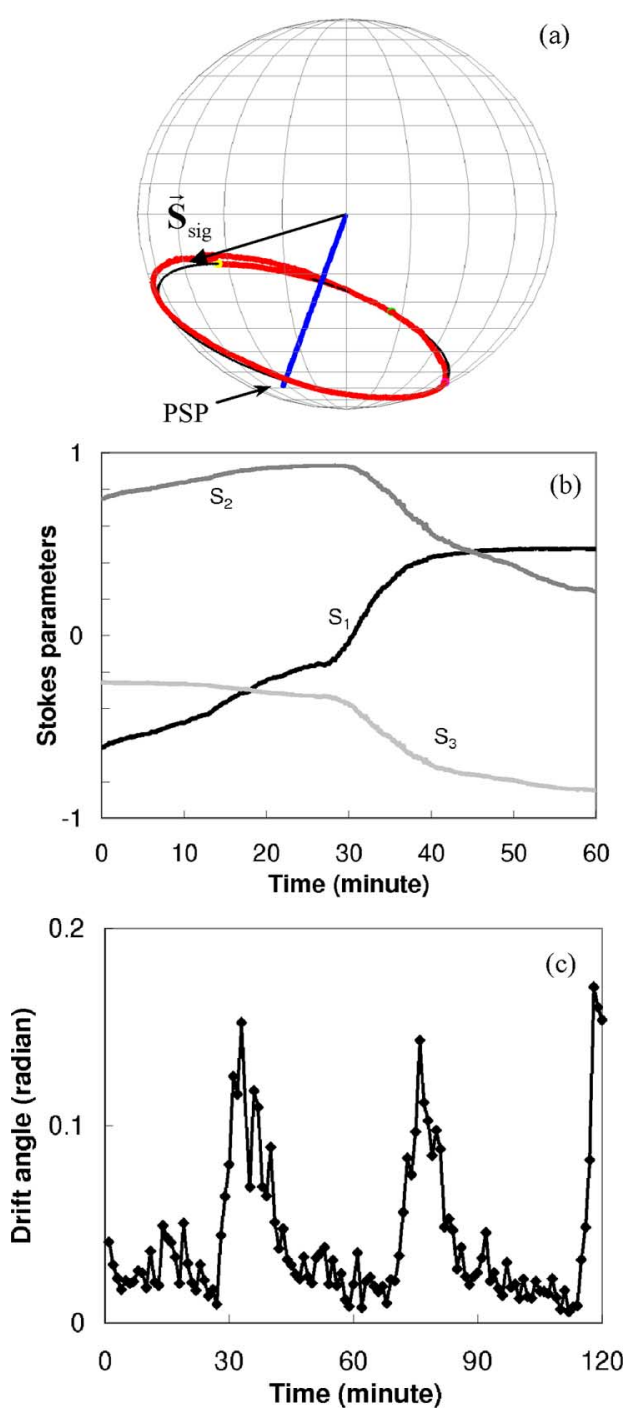

Fig. 6. Drift of the SOP of the signal in an 8-m PM fiber with 10 ps DGD when the fiber is placed in water. (a) Drift of the SOP on the Poincare sphere. (b) Drift of Stokes parameters. (c) Drift angle of the SOP of the signal during 1-min intervals.

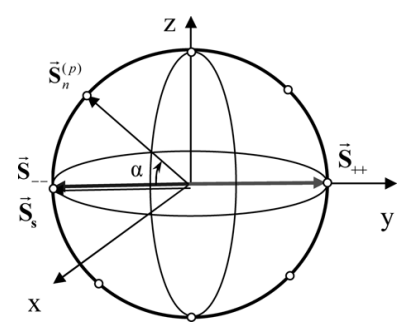

Fig. 7. Experimental setup for a splitting factor of $\left|c_{+}\right|^{2}=0$.

increments in the $y-z$ plane. The eight positions of $\overrightarrow{\mathbf{S}}_{n}^{(p)}$ are indicated by the small circles in Fig. 7. For each angle between $\overrightarrow{\mathbf{S}}_{s}$ and $\overrightarrow{\mathbf{S}}_{n}^{(p)}$, we repeatedly measured the $Q$-factor 70 times and used the mean $Q$-factor to average out the random fluctuations.

In Fig. 8, we show the mean $Q$-factor as a function of the angle $\alpha$ between $\overrightarrow{\mathbf{S}}_{s}$ and $\overrightarrow{\mathbf{S}}_{n}^{(p)}$ in the case that $\left|c_{+}\right|^{2}=0$. For the results in Fig. 8(a) and (b), we set the DOP of the noise to be 0.5
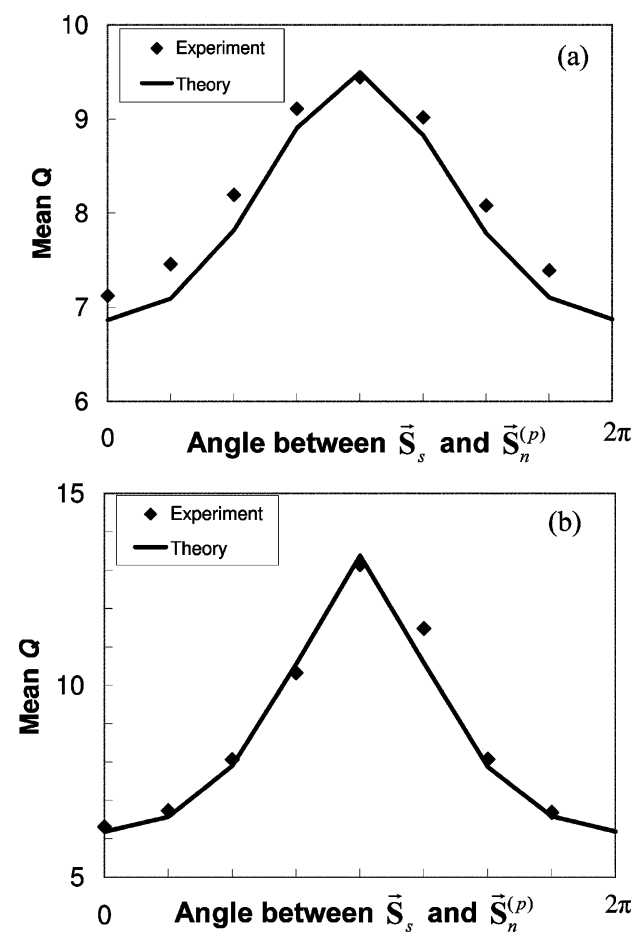

Fig. 8. $Q$-factor as a function of the angle between $\overrightarrow{\mathbf{S}}_{S}$ and $\overrightarrow{\mathbf{S}}_{n}^{(p)}$ for a splitting factor of $\left|c_{+}\right|^{2}=0$. (a) $\mathrm{DOP}_{n}=0.5$. (b) $\operatorname{DOP}_{n}=1.0$.

and 1.0, respectively. The symbols are the measured results and the lines represent the results calculated using (1)-(3) and (15).

From Fig. 8, we see that the measured $Q$-factor is in good agreement with the theoretical results. The mean differences between the measured and calculated $Q$-factors for the eight angles are 0.26 and 0.25 when the DOP of the noise is 0.5 and 1.0, respectively. Just as we saw from the Monte Carlo simulations discussed in Section III, the $Q$-factor attains its minimum and the maximum when the angles between $\overrightarrow{\mathbf{S}}_{s}$ and $\overrightarrow{\mathbf{S}}_{n}^{(p)}$ is 0 and $\pi$, respectively. The magnitude of the variation of the $Q$-factor is also a function of the DOP of the noise. From the calculated results, the variation of the $Q$-factor increases from 2.6 to 7.2 when the DOP of the noise is increased from 0.5 to 1.0.

Next, we consider the case where the signal power is equally split between the two principal modes, i.e., $\left|c_{+}\right|^{2}=0.5$. This case is considered to be the worst case among all launching polarization states because the signal is distorted the most. In this case, the SOP of the signal at the output of the PM fiber drifts slowly over time. As a result, it is difficult to fix the SOP of the signal for a long time during the experiment. Since the SOP of the polarized part of noise is stable, we fixed $\overrightarrow{\mathbf{S}}_{n}^{(p)}$ during the experiment, and let $\overrightarrow{\mathbf{S}}_{s}$ drift slowly on the circle in the $x-z$ plane where the power-splitting ratio is 0.5 , as shown in Fig. 9. For simplicity, we plot the normalized Stokes vector $\overrightarrow{\mathbf{S}}_{s} /\left|\overrightarrow{\mathbf{S}}_{s}\right|$ on the Poincaré sphere.

In Fig. 10, we plot the $Q$-factor as a function of the angle $\tau$ between $\overrightarrow{\mathbf{S}}_{s}$ and $\overrightarrow{\mathbf{S}}_{n}^{(p)}$. For the results shown in Fig. 10(a) and (b), the DOPs of the noise were 0.5 and 1.0, respectively. The diamonds represent the measured $Q$-factor and the circles represent the $Q$-factor calculated using (1)-(3) and (15) using the parameters $\overrightarrow{\mathbf{S}}_{--}, \overrightarrow{\mathbf{S}}_{s}$, and $\overrightarrow{\mathbf{S}}_{n}^{(p)}$, which we measured in the experiments. 


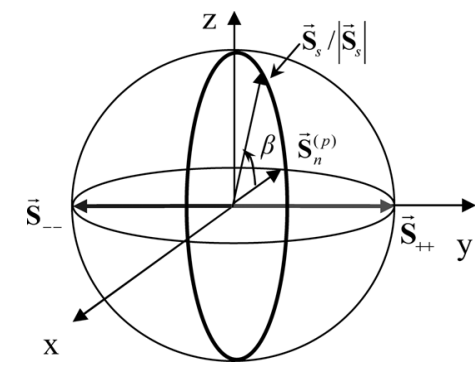

Fig. 9. Experimental setup for a splitting factor of $\left|c_{+}\right|^{2}=0.5$.
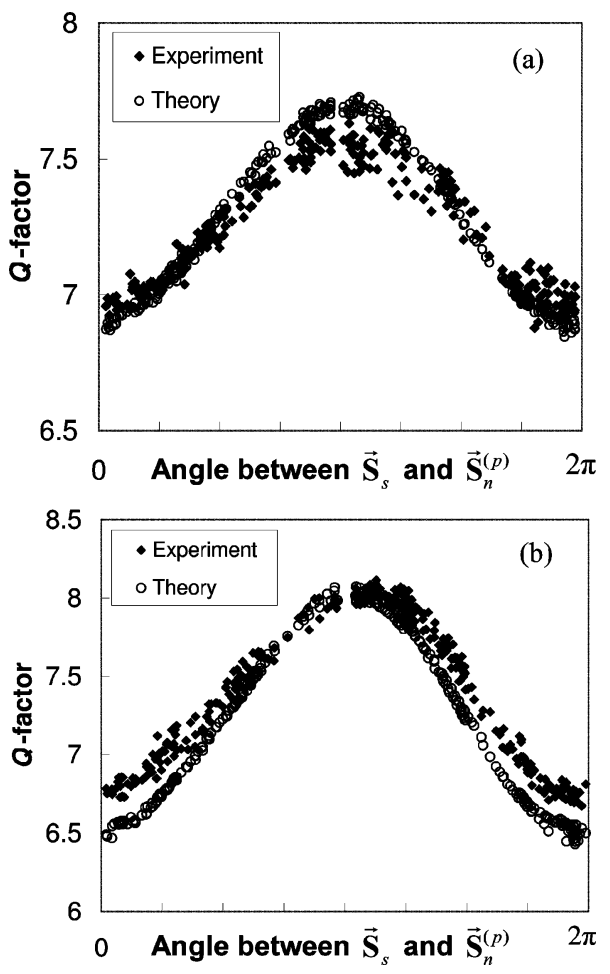

Fig. 10. $Q$-factor as a function of the angle between $\overrightarrow{\mathbf{S}}_{s}$ and $\overrightarrow{\mathbf{S}}_{n}^{(p)}$ for a splitting factor of $\left|c_{+}\right|^{2}=0.5$. (a) $\operatorname{DOP}_{n}=0.5$. (b) $\operatorname{DOP}_{n}=1.0$.

In this case, the $Q$-factor is not averaged over time as in the previous case because $\overrightarrow{\mathbf{S}}_{s}$ is not stable for long enough time. Instead, we plot the correlation between the $Q$-factor and the corresponding angle between $\overrightarrow{\mathbf{S}}_{s}$ and $\overrightarrow{\mathbf{S}}_{n}^{(p)}$. Note that the theoretically computed $Q$-factors do not exactly lie on a smooth curve because of uncertainties in the measurement of $\overrightarrow{\mathbf{S}}_{s}$ and $\overrightarrow{\mathbf{S}}_{n}^{(p)}$. Due to the measurement uncertainty in the $Q$-factor itself and the slow drift of the SOP of the signal during the experiment, the measured $Q$-factor shows larger scattering compared to the calculated $Q$-factor. However, the deviations in most of the $Q$-factors obtained from the experiments and the theory are less than 0.2 in Fig. 10(a) and less than 0.3 in Fig. 10(b), respectively. The level of agreement between the theory and the experiment is very good since the measurement uncertainty of the $Q$-factor itself is around 0.1 in a back-to-back system without PMD when everything is fixed and stable.

\section{LIMITATIONS OF THE THEORY}

In previous sections, we introduced and validated a theoretical receiver model that quantifies the combined effect of firstorder PMD acting on the signal and PDL acting on the noise. However, the higher order PMD of the randomly birefringent fiber in real optical communication systems can also affect the system performance by changing the shape of the pulses and the SOP of the signal. In this section, we use numerical simulations to investigate the accuracy with which the theory predicts the system performance in a more realistic situation in which the signal is impaired by higher order as well as first-order PMD. As in the previous sections, we assume that PDL only acts on the noise and not on the signal.

To address this issue, we replace the PM fiber in the model system shown in Fig. 1 by a segment of randomly birefringent fiber. Rather than gathering statistics over a large collection of random fibers, we generate one-parameter families of fibers with increasing values of DGD. Because of the well-established correlation between DGD and SOPMD [31], this procedure allows us to easily generate fibers with a wide range of SOPMD values.

We used the coarse-step method [32] to generate one-parameter families of fibers as follows. Each fiber in a family consisted of $N$ sections of the PM fiber. For each family, we made a random choice of the polarization rotation between each section. We use the same set of rotations for all fibers in the family. The different fibers in a family were then determined by the choice of a single DGD value for all $N$ sections.

For each fiber in a family, we compared the $Q$-factor calculated using a Monte Carlo simulation to that obtained from the theory. Using this procedure, we determined how large the SOPMD becomes before the receiver model starts to break down. Although our results depend on the choice of polarization rotations used for each family, we expect qualitatively similar results to hold for completely random fibers.

In the Monte Carlo simulations, we computed the DGD and PSP of the fiber at the central frequency of the signal as well as the DOP and SOP of both the signal and noise. These quantities were then used as the values of the input parameters to the theoretical receiver model. For all simulations, we set the DOP of the noise to be 0.5 and the OSNR at the receiver to be $15 \mathrm{~dB}$. For simplicity, we sampled the signal at the center of each bit slot rather than using an automated clock recovery algorithm.

To validate the entire procedure, we first generated a family for which the fibers only had first-order PMD, i.e., we set the number of sections to be $N=1$. In this case, we would expect the agreement between the theory and Monte Carlo simulation to be limited only by the statistical uncertainty in the Monte Carlo simulations. For simplicity, we set the power-splitting ratio to be 0.5 . To obtain the minimum and maximum $Q$-factors for this splitting ratio, we set $\overrightarrow{\mathbf{S}}_{n}^{(p)}=\overrightarrow{\mathbf{S}}_{s}$ and $\overrightarrow{\mathbf{S}}_{n}^{(p)}=-\overrightarrow{\mathbf{S}}_{s}$, respectively. In Fig. 11, we plot the simulated and theoretical $Q$-factors as a function of the DGD. The agreement between 


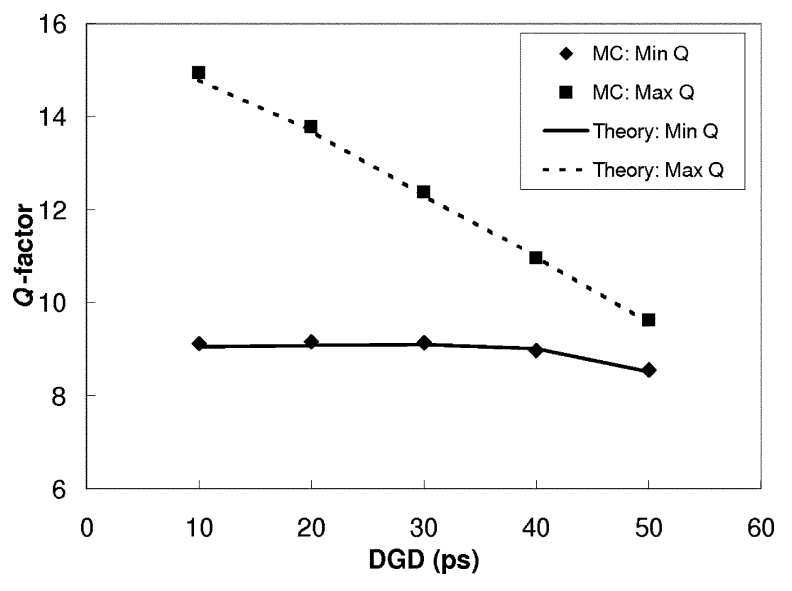

Fig. 11. $Q$-factors obtained from Monte Carlo simulations and formula (15) as a function of DGD when only first-order PMD is included in the fiber model.

TABLE I

VALUES OF DGD AND SOPMD FOR FAMILIES OF FIBERS WITH SMALLER AND LARGER SOPMD TOGETHER WITH THE CORRESPONDING $Q$-FACTORS WHEN THE POLARIZED PART OF NOISE IS IN THE SAME OR OPPOSITE DiRECTION TO THE SOP OF THE SIGNAL AT THE OUTPUT OF THE FIBER

\begin{tabular}{|c|c|c|c|c|c|c|}
\hline \multirow{2}{*}{$\begin{array}{c}\text { Family } \\
\text { of } \\
\text { fibers }\end{array}$} & \multicolumn{2}{|l|}{ PMD in the fiber } & \multicolumn{2}{|c|}{$\begin{array}{c}\text { Q-factors from } \\
\text { Monte Carlo } \\
\text { simulations }\end{array}$} & \multicolumn{2}{|c|}{$\begin{array}{c}\text { Q-factors from } \\
\text { the theory }\end{array}$} \\
\cline { 2 - 7 } & $\begin{array}{c}\text { DGD } \\
\text { (ps) }\end{array}$ & $\begin{array}{c}\text { SOPMD } \\
\left.\text { (ps }^{2}\right)\end{array}$ & Min. Q & Max. Q & Min. Q & Max. Q \\
\hline \multirow{4}{*}{$\begin{array}{c}\text { Smaller } \\
\text { SOPMD }\end{array}$} & 10.25 & 65 & 9.07 & 14.86 & 9.05 & 14.89 \\
\cline { 2 - 7 } & 20.4 & 260 & 9.00 & 13.88 & 9.02 & 14.03 \\
\cline { 2 - 7 } & 30.9 & 370 & 8.91 & 12.63 & 8.71 & 13.24 \\
\cline { 2 - 7 } & 39.92 & 415 & 8.75 & 12.57 & 7.84 & 15.32 \\
\hline \multirow{4}{*}{ Larger } & 49.51 & 988 & 8.01 & 11.97 & 7.04 & 16.92 \\
\cline { 2 - 7 } SOPMD & 10.66 & 186 & 9.02 & 14.91 & 8.98 & 15.09 \\
\cline { 2 - 7 } & 20.2 & 670 & 8.82 & 14.04 & 9.04 & 13.67 \\
\cline { 2 - 7 } & 30.61 & 1810 & 8.12 & 12.62 & 9.28 & 11.88 \\
\cline { 2 - 7 } & 41.2 & 2460 & 7.93 & 11.76 & 7.30 & 20.16 \\
\hline
\end{tabular}

the theory and the Monte Carlo simulations is excellent, even for DGD values as large as $50 \mathrm{ps}$.

Next, to investigate the limitations of the theoretical model in the presence of higher order PMD, we applied the procedure described before to generate families of fibers with $N=15$ sections. We generated a smaller SOPMD and a larger SOPMD family, each of which consisted of five fibers with DGD values ranging from 10 to $50 \mathrm{ps}$. The values of the DGD and SOPMD of these fibers are given in Table I.

For the smaller SOPMD family, the values of SOPMD were between about $45 \%$ and $110 \%$ of the expected value, i.e., $\langle\mathrm{SOPMD}\rangle=\langle\mathrm{DGD}\rangle^{2} / \sqrt{3}$ of the SOPMD, whereas for the larger SOPMD family, the majority of SOPMD values were about three times the expected value. We chose the SOP of the polarized part of the noise to be in the same (opposite) direction in Stokes space to the average SOP of the signal at the output of the fiber, which enabled us to approximate the minimum (respectively maximum) value of the $Q$-factor. In Table I, we show these approximations to the minimum and maximum $Q$-factors computed using both Monte Carlo simulations and using the theoretical model. For both families of fibers, the absolute error between the minimum $Q$-factor obtained using Monte Carlo simulations and with the theoretical model is less than 1.5 , even for very large value of SOPMD. However, the error in maximum $Q$-factor obtained from the theory can be extremely large when the SOPMD is on the order of $1000 \mathrm{ps}^{2}$ or larger. Moreover, we find that for similar DGD values, the higher the SOPMD, the larger the error in the theoretically computed $Q$-factor. For these two families of fibers, we require SOPMD values on the order of $300 \mathrm{ps}^{2}$ or less to ensure that the error in the theoretical $Q$-factor is less than 0.5 for both the minimum and maximum $Q$-factors.

\section{CONCLUSION}

In conclusion, we systematically investigated the combined effect of first-order PMD and the PDL on the system performance when PMD induces significant distortion and depolarization of the signal and PDL causes the noise to become partially polarized. We derived a formula for the variance of the electric current in the marks and the spaces that is due to the beating between a depolarized signal and partially polarized noise. This formula correctly accounts for the optical pulse shape and the shapes of the electrical and the optical filters. We validated this formula by comparing the $Q$-factor obtained from the theory to that obtained from numerical simulations and from experiments, and we obtained very good agreement. The receiver model that we have introduced and experimentally validated in this paper can be applied to systems in which neither waveform distortions due to PDL nor higher order PMD play a statistically significant role in determining the distribution of the power penalty (or the $Q$-factor). These conditions are expected to hold in many realistic systems, and specifically for those in which the average PDL of the system does not exceed $5 \mathrm{~dB}$ [11] and the high-order PMD is on the order of $300 \mathrm{ps}^{2}$ or less. For such systems, the model could in future be used to quantify the sources of variation in the $Q$-factor. To illustrate the potential applications of the model, we showed that the BER varies from about $10^{-26}$ to $10^{-14}$ due to variations in the angle between the polarization states of the signal and noise when the DOP of the noise is 0.5 and the PMD power-splitting ratio takes on the worst-case value of 0.5 .

In the future, the model could be more systematically applied to compute the probability distribution of the $Q$-factor due to random variations in the polarization effects. Such a $Q$-distribution could help system designers allocate a power margin for polarization effects in systems for which PDL-induced waveform distortions and high-order PMD are insignificant. Finally, the theoretical and experimental results presented here establish a solid foundation for more general future receiver models in which both signal and noise are impaired by distributed PDL and PMD. 


\section{REFERENCES}

[1] F. Bruyère and O. Audouin, "Penalties in long-haul optical amplifier systems due to polarization dependent loss and gain," IEEE Photon. Technol. Lett., vol. 6, no. 5, pp. 654-656, May 1994.

[2] J. Zhou and M. J. O'Mahony, "Optical transmission system penalties due to fiber polarization mode dispersion," IEEE Photon. Technol. Lett., vol. 6, no. 10, pp. 1265-1267, Oct. 1994.

[3] C. R. Menyuk, D. Wang, and A. N. Pilipetskii, "Repolarization of polarization-scrambled optical signals due to polarization dependent loss," IEEE Photon. Technol. Lett., vol. 9, no. 9, pp. 1247-1249, Sep. 1997.

[4] L.-S. Yan, Q. Yu, Y. Xie, and A. E. Willner, "Experimental demonstration of the system performance degradation due to the combined effect of polarization-dependent loss with polarization-mode dispersion," IEEE Photon. Technol. Lett., vol. 14, no. 2, pp. 224-226, Feb. 2002.

[5] Y. Sun, I. T. Lima, Jr., A. O. Lima, H. Jiao, J. Zweck, L. Yan, G. M. Carter, and C. R. Menyuk, "System performance variations due to partially polarized noise in a receiver," IEEE Photon. Technol. Lett., vol. 15 , no. 11, pp. 1648-1650, Nov. 2003.

[6] A. Mecozzi and M. Shtaif, "The statistics of polarization-dependent loss in optical communication systems," IEEE Photon. Technol. Lett., vol. 14, no. 3, pp. 313-315, Mar. 2002.

[7] C. Xie and L. F. Mollenauer, "Performance degradation induced by polarization-dependent loss in optical fiber transmission systems with and without polarization-mode dispersion," J. Lightw. Technol., vol. 21, no. 9, pp. 1953-1957, Sep. 2003.

[8] M. Shtaif and A. Mecozzi, "Polarization-dependent loss and its effect on the signal-to-noise ratio in fiber-optic systems," IEEE Photon. Technol. Lett., vol. 16, no. 2, pp. 671-673, Feb. 2004.

[9] D. Wang and C. R. Menyuk, "Calculation of penalties due to polarization effects in a long-haul WDM system using a Stokes parameter model," J. Lightw. Technol., vol. 19, no. 4, pp. 487-494, Apr. 2001.

[10] B. Huttner, C. Geiser, and N. Gisin, "Polarization-induced distortion in optical fiber networks with polarization mode dispersion and polarization-dependent losses," IEEE J. Sel. Topics Quantum Electron., vol. 6, no. 2, pp. 317-329, Mar./Apr. 2000.

[11] M. Shtaif and O. Rosenberg, "Polarization-dependent loss as a waveform-distorting mechanism and its effect on fiber-optical systems," $J$. Lightw. Technol., vol. 23, no. 2, pp. 923-930, Feb. 2005.

[12] N. Y. Kim, D. Lee, H. Yoon, J. Park, and N. Park, "Limitation of PMD compensation due to polarization dependent loss in high-speed optical transmission links," IEEE Photon. Technol. Lett., vol. 14, no. 1, pp. 104-106, Jan. 2002.

[13] I. T. Lima, Jr., A. O. Lima, Y. Sun, H. Jiao, J. Zweck, C. R. Menyuk, and G. M. Carter, "A receiver model for optical fiber communication systems with arbitrarily polarized noise," J. Lightw. Technol., vol. 23, no. 3, pp. 1478-1490, Mar. 2005.

[14] J. L. Rebola and A. V. T. Cartaxo, "Performance evaluation of optically preamplified receivers with partially polarized noise and arbitrary optical filtering: A rigorous approach," Proc. Inst. Electr. Eng. Optoelectron., vol. 152, pp. 251-262, 2005.

[15] L. Chen, Z. Zhang, and X. Bao, "Combined PMD-PDL effects on BERs in simplified optical systems: An analytical approach," Opt. Exp., vol. 15, pp. 2106-2119, 2007.

[16] Z. Zhang, L. Chen, and X. Bao, "Accurate BER evaluation for lumped DPSK and OOK systems with PMD and PDL," Opt. Exp., vol. 15, pp. 9418-9433, 2007.

[17] D. Marcuse, "Derivation of analytical expressions for the bit-error probability in lightwave systems with optical amplifiers," J. Lightw. Technol., vol. 8, no. 12, pp. 1816-1823, Dec. 1990.

[18] P. A. Humblet and M. Azizoğlu, "On the bit error rate of lightwave systems with optical amplifiers," J. Lightw. Technol., vol. 9, no. 11, pp. 1576-1582, Nov. 1991.

[19] E. Forestieri, "Evaluating the error probability in lightwave systems with chromatic dispersion, arbitrary pulse shape and pre-and postdetection filtering," J. Lightw. Technol., vol. 18, no. 11, pp. 1493-1503, Nov. 2000.

[20] P. J. Winzer, M. Pfennigbauer, M. M. Strasser, and W. R. Leeb, "Optimum filter bandwidth for optically preamplified NRZ receivers," $J$. Lightw. Technol., vol. 19, no. 9, pp. 1263-1273, Sep. 2001.
[21] J. L. Rebola and A. V. T. Cartaxo, "Power penalty assessment in optically preamplified receivers with arbitrary optical filtering and signaldependent noise dominance," J. Lightw. Technol., vol. 20, no. 3, pp. 401-408, Mar. 2002.

[22] M. Pfennigbauer, M. M. Strasser, M. Pauer, and P. J. Winzer, "Dependence of optically preamplified receiver sensitivity on optical and electrical filter bandwidths-Measurement and simulation," IEEE Photon. Technol. Lett., vol. 14, no. 6, pp. 831-833, Jun. 2002.

[23] J. Zweck and C. R. Menyuk, "Validity of the additive white Gaussian noise model for quasi-linear long-haul return-to-zero optical fiber communications systems," J. Lightw. Technol., 2009, to be published.

[24] I. T. Lima, Jr., and A. M. Lima, "Computation of the probability of power penalty and Q-penalty outages due to PMD," in Proc. LEOS Annu. Meeting, 2003, pp. 316-317, Paper TuQ1.

[25] W. Xi, T. Adali, and J. Zweck, "A MAP equalizer for the optical communications channel," J. Lightw. Technol., vol. 23, no. 12, pp. 3989-3996, Dec. 2005.

[26] A. Mecozzi and M. Shtaif, "Signal-to-noise-ratio degradation caused by polarization-dependent loss and the effect of dynamic gain equalization," J. Lightw. Technol., vol. 22, no. 8, pp. 1856-1871, Aug. 2004.

[27] J. P. Gordon and H. Kogelnik, "PMD fundamentals: Polarization mode dispersion in optical fibers," Proc. Nat. Acad. Sci. USA, vol. 97, pp. 4541-4550, 2000.

[28] H. Xu, H. Jiao, L. Yan, and G. M. Carter, "Measurement of distributions of differential group delay in a recirculating loop with and without loop-synchronous scrambling," IEEE Photon. Technol. Lett., vol. 16, no. 7, pp. 1691-1693, Jul. 2004.

[29] N. Bergano, F. W. Kerfoot, and C. R. Davidson, "Margin measurements in optical amplifier systems," IEEE Photon. Technol. Lett., vol. 5, no. 3, pp. 304-306, Mar. 1993.

[30] M. Karlsson, J. Brentel, and P. A. Andrekson, "Long-term measurement of PMD and polarization drift in installed fibers," J. Lightw. Technol., vol. 18, no. 7, pp. 941-951, Jul. 2000.

[31] E. Ibragimov, G. Shtengel, and S. Suh, "Statistical correlation between first and second-order PMD," J. Lightw. Technol., vol. 20, no. 4, pp. 586-590, Apr. 2002.

[32] D. Marcuse, C. R. Menyuk, and P. K. A. Wai, "Application of the Manakov-PMD equation to studies of signal propagation in optical fibers with randomly varying birefringence," J. Lightw. Technol., vol. 15, no. 9, pp. 1735-1746, Sep. 1997.

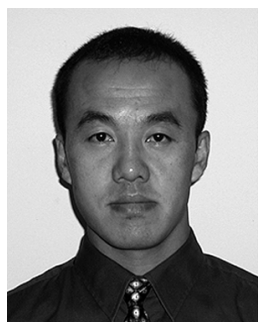

Hua Jiao (M'99) received the B.S. and M.S. degrees from Peking University, Beijing, China, in 1996 and 1999, respectively, and the Ph.D. degree from the University of Maryland Baltimore County, Baltimore, in 2007.

In 2006, he joined AT\&T Laboratories, San Ramon, CA, where he was involved in the field of xDSL technologies. In 2008, he joined Ciena, Linthicum, MD, where he is engaged in research on tunable lasers, modulators, and plug-in optics.

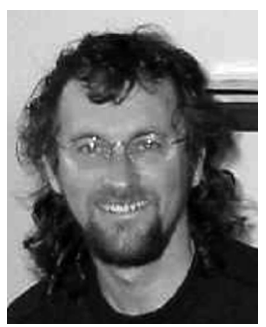

John Zweck received the B.Sc. degree (with honors) from the University of Adelaide, Adelaide, S.A., Australia, in 1988, and the Ph.D. degree in mathematics from Rice University, Houston, TX, in 1993.

$\mathrm{He}$ was engaged in research on differential geometry, human and computer vision, computational anatomy, and modeling of optical systems. Since 2006, he has been an Associate Professor of mathematics and statistics and an Affiliate Associate Professor of computer science and electrical engineering at the University of Maryland Baltimore County (UMBC), Baltimore, where he was a Research Associate in the Department of Computer Science and Electrical Engineering from 2000 to 2003, and an Assistant Professor in the Department of Mathematics and Statistics from 2003 to 2006. His current research interests include modeling of optical fiber communications systems, short-pulse lasers, and photoacoustic spectroscopy sensors. He is the Lead Developer of software PhoSSiL, the Photonics Systems Simulator Library.

Dr. Zweck is a member of the Society for Industrial and Applied Mathematics. 
Li Yan (S'84-M'87) received the B.S. degree in physics from the University of Science and Technology of China, Hefei, China, in 1982, and the M.S. and $\mathrm{Ph}$.D. degrees in physics from the University of Maryland, College Park, in 1986 and 1989 , respectively.

In 1990, he joined the Faculty of Electrical Engineering, University of Maryland Baltimore County (UMBC), Baltimore, where he is currently a Professor in the Department of Computer Science and Electrical Engineering. His current research interests include ultrafast optics, solid-state lasers, optical communications, and nonlinear optics.

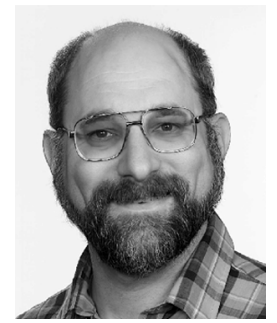

Curtis R. Menyuk (SM'88-F'98) was born in March 26, 1954. He received the B.S. and M.S. degrees from Massachusetts Institute of Technology (MIT), Cambridge, in 1976, and the Ph.D. degree from the University of California Los Angeles (UCLA), Los Angeles, in 1981.

He was a Research Associate at the University of Maryland, College Park, and at Science Applications International Corporation, McLean, VA. In 1986, he became an Associate Professor in the Department of Electrical Engineering, University of Maryland Baltimore County (UMBC), Baltimore, where he was promoted to a Professor in 1993 and was a former Presidential Research Professor. He was the founding member of the Department of Electrical Engineering, UMBC. From Fall 1996 until Fall 2002, he was on partial leave from UMBC. From 1996 to 2001, he worked part time at the Department of Defense, DoD Laboratory for Telecommunications Sciences, Adelphi, MD, where he codirected the Optical Networking Program from 1999 to 2001. During 2001-2002, he was a Chief Scientist at PhotonEx Corporation. He is engaged in research on theoretical and computational studies of lasers, nonlinear optics, and fiber optic communications for the last 18 years. He has authored or coauthored more than 200 archival journal publications as well as numerous other publications and presentations, and has also edited three books.

Prof. Menyuk is a member of the Society for Industrial and Applied Mathematics and the American Physical Society. He is a Fellow of the Optical Society of America.

Gary M. Carter, photograph and biography not available at the time of publication. 